\section{Normal variant striate calcification perpendicular to the lateral ventricles}

\section{Ralph Drosten MB BCh, FCRad (D)}

Department of Thoracic Imaging Brigham and Women's Hospital Boston, USA

\section{Case presentation}

A 43-year-old man presented to his doctor after his second grand mal seizure. He was found to be entirely normal on clinical and neurological examination, laboratory testing and

\section{Discussion}

Striate calcification perpendicular

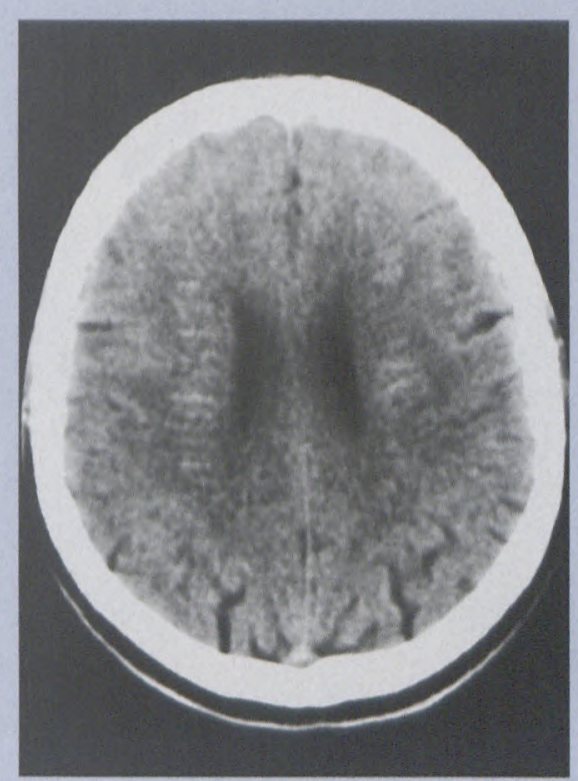

Fig.1. Pre-contrast axial CT of the brain at the level of the lateral ventricles showing striate calcifications perpendicular to the lateral ventricles in the deep white matter of the corona radiata.

to the lateral ventricles is a recognised normal variant and has no known pathological or clinical relevance. 